\title{
Effect of a Collaboration Between a Health Plan, Oncology Practice, and Comprehensive Genomic Profiling Company from the Payer Perspective
}

\author{
Mitchell Reitsma, BA, MBA; John Fox, MD; Pierre Vanden Borre, PhD; Matthew Cavanaugh, BSN, RN; \\ Yakov Chudnovsky, PhD; Rachel L. Erlich, PhD; Thomas E. Gribbin, MD; and Rachel Anhorn, PharmD
}

\begin{abstract}
BACKGROUND: Comprehensive genomic profiling (CGP) is a next-generation sequencing-based methodology that detects 4 classes of genomic alterations, as well as gene signature biomarkers such as microsatellite instability and tumor mutational burden. In the context of precision oncology, CGP can help to direct treatment to genomically matched therapies.

OBJECTIVE: To describe the results of a 3-year observational analysis of patients undergoing testing with CGP assays (either Foundation0ne or Foundation One Heme) at a community oncology practice after a regional health plan implemented a medical policy that enabled coverage of CGP.

METHODS: A retrospective analysis of medical records was completed at the oncology practice from November 2013 to January 2017; this date range was chosen to coincide with the regional health plan's medical policy implementation of CGP. The medical policy provided coverage of CGP for patients with advanced solid and hematologic cancers. A medical record review assessed all previous and current molecular test results, matched therapy or clinical trial enrollment, and clinical outcomes (clinical benefit or disease progression). The potential cost diversion, from payer to study sponsor, for patients who enrolled in clinical trials was explored.
\end{abstract}

RESULTS: There were 96 patients in the community oncology practice who received CGP over the 3-year period, 86 of whom had clinically relevant genomic alterations. Of the 86,15 patients were treated with genomically matched therapy, and 6 patients enrolled in clinical trials based on CGP results. In a subset of 32 patients who previously underwent conventional testing, most (84\%) had clinically relevant genomic alterations detected by CGP that conventional testing did not identify, and a portion of these patients subsequently received treatment based on the CGP results. In the separate cost diversion analysis of 20 patients who enrolled in phase 1 clinical trials, an estimated $\$ 25,000$ per-patient cost-benefit may have been accrued to the payer.

CONCLUSIONS: This observational analysis characterized the use of CGP in a large community oncology practice among a group of patients insured by a regional health plan. Clinical trial enrollment was facilitated by CGP use in the community setting and may have contributed to cost diversion from the payer to study sponsors.

J Manag Care Spec Pharm. 2019;25(5):601-11

Copyright $\odot 2019$, Academy of Managed Care Pharmacy. All rights reserved.

\section{What is already known about this subject}

Conventional molecular diagnostic testing is often used in clinical practice; however, up to $30 \%$ of tests fail because of insufficient biopsy material, insufficient DNA, or failed library preparation. Comprehensive genomic profiling (CGP) is a next-generation sequencing-based methodology that can help to direct treatment to genomically matched therapies with efficient tissue use.

Research supporting the clinical utility of CGP is extensive, but there is a need for data in the real-world, community-based setting.

\section{What this study adds}

Of patients who received CGP in a community oncology setting, this method of testing identified clinically relevant genomic alterations in most patients, and just under one quarter were treated with matched therapy or enrolled into a clinical trial based on test results.

A preliminary cost diversion analysis suggests that there is a cost-benefit associated with clinical trial enrollment, which is facilitated by CGP.

The observational analysis of medical records provides a proof of concept for covering CGP and integrating it into clinical practice.

$\mathrm{P}$ recision medicine-based testing to identify biomarkerdriven matched therapies in the oncology setting can include 3 general types of tests: single-gene and multigene hotspot panels (both considered to be conventional molecular diagnostic testing), as well as comprehensive genomic profiling (CGP) assays. A CGP assay detects 4 classes of genomic alterations (substitutions, insertion and deletion alterations, copy number alterations, and rearrangements) across a comprehensive set of genes relevant in cancer, as well as genomic signatures, such as microsatellite instability and tumor mutational burden (TMB) in a single assay and report., ${ }^{1,2}$ Conventional testing analyzes limited classes of alterations in a restricted set of genes or regions of genes. Although conventional molecular diagnostic testing is often used in clinical practice, up to 30\% of tests fail because of insufficient biopsy material, insufficient DNA, or failed library preparation. ${ }^{3}$ By contrast, CGP allows a considerable amount of molecular profile data to be 
identified with efficient tissue use. CGP results include alterations and biomarkers that can be used to direct on-label treatment with targeted therapies approved by the U.S. Food and Drug Administration (FDA), as well as alterations and biomarkers that can guide the use of other targeted therapies and immunotherapies, including those available through clinical trial programs. ${ }^{1,2,4,5}$

Payer reluctance to cover CGP may include concerns about off-label drug use, cost per test, lack of test-specific guideline inclusion, and experimental/investigational or medically unnecessary designation of broad panel tests. However, emerging real-world and clinical trial data confirm the clinical utility of genomically matched therapy as demonstrated by improved outcomes compared with unmatched therapy across a wide range of cancers. ${ }^{6-13}$ Despite this, many patients who could benefit from biomarker-driven therapy selection do not receive even basic, guideline-recommended, single-gene testing before starting treatment, presumably because of the clinical and logistical challenges of using a gene-by-gene testing approach, including clinician judgment and tissue insufficiency. ${ }^{14} \mathrm{~A}$ comprehensive approach to genomic profiling can facilitate the use of matched therapies regardless of tumor type. ., $12,13,15-18^{-1}$

The possible clinical and economic benefits of CGP for increasing clinical trial enrollment may be substantial for patients in community settings. Clinical trial enrollment is encouraged by national organizations, such as the American Society of Clinical Oncology, the National Comprehensive Cancer Network (NCCN), and the Cancer Moonshot Initiative. ${ }^{19-21}$ However, trial participation remains low outside of academic centers. ${ }^{22}$ The lack of on-site next-generation sequencing (NGS) pathology laboratories in community clinics, an important component of providing CGP, is a considerable barrier to identifying and enrolling patients in clinical trials for genomically matched and biomarker-driven therapies. Offering (or enabling access to) CGP through a central laboratory may, therefore, facilitate clinical trial enrollment-which has been associated with improved patient survival when compared with care outside of a clinical trial2 ${ }^{22}$-in the community setting. Because drug costs for patients enrolled in clinical trials are generally diverted to clinical trial sponsors, a possible area of investigation is whether facilitating such enrollment can confer economic benefits to payers. Further investigations into the clinical and economic outcomes of CGP-guided therapy in community settings are, therefore, warranted.

The present observational review of medical records assessed the real-world use of 2 assays, FoundationOne and FoundationOne Heme (Foundation Medicine, Cambridge, MA). Both assays provide clinically and analytically validated CGP that is certified by the Clinical Laboratory Improvement Amendments and the New York Department of Health, the former directed at solid tumors and the latter directed at hematologic malignancies and sarcomas. ${ }^{2,23}$ Both assays were commercially available, facilitated by a collaboration between various stakeholders including a health plan and a community oncology practice. In particular, this article describes the findings of a 3-year review of patients' medical records following the health plan's adoption of a medical policy covering CGP. The observational analysis also provides exploratory data on potential cost savings for payers following clinical trial enrollment.

\section{Methods}

\section{Study Design and Data Source}

This was a retrospective review of medical records of patients with cancer treated at a single-center practice. Review/approval by an institutional review board was not required for this study, since it used de-identified data with authorization from Priority Health. CGP data from patients' tumor samples were obtained using the FoundationOne or FoundationOne Heme assays and included in this analysis. Patients received CGP as part of a collaboration between a community oncology practice that provides specialty care and clinical trial coordination in the western Michigan region (Cancer and Hematology Centers of West Michigan [CHCWM]); a health plan (Priority Health, a nonprofit, independent insurance provider that covers patients enrolled in Medicare, Medicaid, and commercial plans); and a molecular insights company (Foundation Medicine). The health plan provides insurance coverage for approximately $30 \%$ of the oncology practice's patients, and the health plan and practice have a 20-year collaboration including oncology care initiatives. . $^{4,25}$

Beginning in 2013, the provider engaged the health plan in a dialogue about coverage of CGP; the health plan then determined coverage and published a medical policy titled "MultiMarker Tumor Panels" (Appendix A, available in online article) that required diagnosis in 1 of 7 indications, minimal life expectancy of 6 months, and Eastern Cooperative Oncology Group (ECOG) performance status of 0,1 , or $2 .{ }^{26}$ The rationale for covering these indications is as follows:

- New-onset stage IV non-small cell lung cancer (NSCLC): The health plan already covered targeted therapies that are approved by the FDA and recommended by the NCCN Clinical Practice Guidelines in Oncology. NSCLC has the greatest number of known actionable alterations of all cancer types. A planned policy update, pending at the time of this writing, will expand the eligible population to also include stage IIIb patients in addition to stage IV patients.

- New-onset cancer of unknown primary: Such cancers have diverse molecular profiles and repeated single-alteration tests increase costs. ${ }^{27,28}$ These cancers also have very limited treatment options as well as a prolonged time to diagnosis (approximately twice as long as cancers with a known primary site) and subsequent delay in treatment. ${ }^{27-29}$

- New-onset hematologic malignancies with high frequencies of actionable mutations or limited options: Hematologic cancers, 
Effect of a Collaboration Between a Health Plan, Oncology Practice, and Comprehensive Genomic Profiling Company from the Payer Perspective

\begin{tabular}{|c|c|}
\hline Characteristic & $\begin{array}{l}\text { All Patients } \\
(\mathrm{N}=96)\end{array}$ \\
\hline Median age, y (range) & $63.5(32-87)$ \\
\hline Female sex & 46 \\
\hline \multicolumn{2}{|l|}{ Geographic location } \\
\hline Large central metropolitan area & 51 \\
\hline Large fringe metropolitan area & 25 \\
\hline Medium metropolitan area & 1 \\
\hline Small metropolitan area & 6 \\
\hline Micropolitan area & 11 \\
\hline Noncore area & 2 \\
\hline Median lines of pre-CGP therapy completed (range) & $0(0-6)$ \\
\hline 0 & 51 \\
\hline 1 & 20 \\
\hline 2 & 14 \\
\hline$\geq 3$ & 11 \\
\hline \multicolumn{2}{|l|}{ Tumor type } \\
\hline Non-small cell lung carcinoma & 36 \\
\hline Colorectal carcinoma & 13 \\
\hline Breast carcinoma & 8 \\
\hline Urothelial/bladder carcinoma & 7 \\
\hline Carcinoma of unknown primary & 5 \\
\hline Sarcoma & 5 \\
\hline Hematological malignancy & 4 \\
\hline Melanoma & 4 \\
\hline Pancreatobiliary carcinoma & 4 \\
\hline All others & 10 \\
\hline \multicolumn{2}{|l|}{ Disease stage } \\
\hline III & 6 \\
\hline IV & 78 \\
\hline Other & 12 \\
\hline \multicolumn{2}{|l|}{ ECOG status } \\
\hline 0 & 21 \\
\hline 1 & 43 \\
\hline 2 & 25 \\
\hline 3 & 3 \\
\hline Missing data & 4 \\
\hline
\end{tabular}

Note: All values are number of patients unless otherwise noted.

CGP = comprehensive genomic profiling; ECOG = Eastern Collaborative Oncology Group

in general, have high frequencies of actionable mutations, and CGP may provide optimal clinical management by informing diagnosis, classification, risk stratification, and treatment decisions. ${ }^{30}$

- The remaining indications were intended to identify patients for clinical trials.

Foundation Medicine performed the CGP assay and interpreted results at a centralized facility in Cambridge, MA. Monthly multidisciplinary molecular tumor board conferences with senior delegates from CHCWM and Foundation Medicine convened to discuss the select cases' CGP results, medical

\section{TABLE 2 CGP Testing and Treatment Patterns}

\begin{tabular}{|c|c|}
\hline & $\begin{array}{l}\text { All Patients } \\
(\mathrm{N}=96)\end{array}$ \\
\hline CGP test successfully reported results & 95 \\
\hline $\begin{array}{l}\text { Cases with clinically relevant genomic alteration detected } \\
\text { (FDA-approved therapy and clinical trials) }\end{array}$ & 86 \\
\hline$\geq 1$ CGP-matched FDA-approved therapy ${ }^{\mathrm{a}}$ & 76 \\
\hline $\begin{array}{l}\text { CGP-matched therapy approved for the treatment of } \\
\text { the patient's tumor type }\end{array}$ & 41 \\
\hline $\begin{array}{l}\text { Only CGP-matched therapy approved for the treat- } \\
\text { ment of another tumor type (off-label)a }\end{array}$ & 35 \\
\hline $\begin{array}{l}\geq 1 \text { clinical trial associated with a CGP-detected genomic } \\
\text { alteration }\end{array}$ & 84 \\
\hline No clinically relevant genomic alterations detected & 9 \\
\hline Clinical trial enrollment & 9 \\
\hline CGP-directed ${ }^{b}$ & 6 \\
\hline Not CGP-directed ${ }^{b}$ & 3 \\
\hline Treated following CGP & 70 \\
\hline Targeted therapy or immunotherapy & 33 \\
\hline CGP-matched therapy & 15 \\
\hline Nontargeted therapy (including radiation therapy) & 37 \\
\hline Declined treatment & 24 \\
\hline Unknown & 2 \\
\hline \multicolumn{2}{|l|}{$\begin{array}{l}\text { Note: All values are presented as number of patients. } \\
\text { a Including sensitive or contraindicated therapies. } \\
{ }^{b} \text { According to treating physician. }\end{array}$} \\
\hline
\end{tabular}

history, and potential effect on clinical management. Notably, the community oncology practice included enrollment in clinical trials as a management recommendation for appropriate patients based on CGP or other medical ontology/history. Patients were enrolled in the following trials: phase 2 and 3 trials through the Cancer Research Consortium of Western Michigan, a National Cancer Institute Community Oncology Research Program; phase 3 trials through the practice; and phase 1 trials through South Texas Accelerated Research Therapeutics Midwest, a practice-affiliated phase 1 program (started in 2016).

\section{Data Analysis and Outcome Measures}

Medical records of the cohort of patients, as described previously, who had received CGP during the collaboration period (2013-2017) and who had at least 12 months of follow-up data were analyzed. Data characterizing the patients, the placement of CGP in their therapy, and the alignment of posttest treatment with CGP results were extracted. Clinical outcomes (clinical benefit or disease progression), relationship of clinical and disease characteristics to the requirements in the CGP medical policy, CGP and previous molecular diagnostic test results, overall survival (OS), and clinical trial enrollment were summarized. 
Effect of a Collaboration Between a Health Plan, Oncology Practice, and Comprehensive Genomic Profiling Company from the Payer Perspective

\section{TABLE 3 CGP-Directed Test Treatment Options and Outcomes}

\begin{tabular}{|c|c|c|c|c|c|}
\hline & $\begin{array}{l}\text { All Patients } \\
(\mathrm{N}=95)^{\mathrm{a}}\end{array}$ & $\begin{array}{l}\text { Non-CGP-Directed } \\
\text { Clinical Trial } \\
\text { Enrollment }(n=3)\end{array}$ & $\begin{array}{l}\text { CGP-Directed } \\
\text { Clinical Trial } \\
\text { Enrollment }(n=6)\end{array}$ & $\begin{array}{l}\text { Treated with a } \\
\text { CGP-Matched } \\
\text { Targeted Therapy } \\
\text { or Immunotherapy } \\
\quad(\mathbf{n}=15)\end{array}$ & $\begin{array}{c}\text { Not Enrolled in } \\
\text { Clinical Trial and } \\
\text { Did Not Receive } \\
\text { CGP-Matched } \\
\text { Targeted Therapy } \\
\text { or Immunotherapy } \\
(\mathbf{n}=71)\end{array}$ \\
\hline \multicolumn{6}{|l|}{ Available treatment options } \\
\hline No associated therapies or clinical trials & 9 & 2 & 0 & 0 & 7 \\
\hline On-label FDA-approved & 34 & 0 & 4 & 10 & 20 \\
\hline Off-label FDA-approved only & 39 & 1 & 1 & 4 & 33 \\
\hline Clinical trial only & 13 & 0 & 1 & 1 & 11 \\
\hline \multicolumn{6}{|l|}{ Treatment response } \\
\hline Clinical benefit & 35 & 1 & 3 & 10 & 21 \\
\hline Disease progression & 57 & 2 & 3 & 5 & 47 \\
\hline Not reported & 3 & 0 & 0 & 0 & 3 \\
\hline Patients with available survival data, $\mathrm{n}$ & 67 & \multicolumn{2}{|c|}{$7 \mathrm{~b}$} & 10 & 50 \\
\hline Median OS, months (range) & $4.8(0-31)$ & \multicolumn{2}{|c|}{$4.5(2.3-20.6)$} & $9.5(1.1-24.2)$ & $4.6(0-30.9)$ \\
\hline \multicolumn{6}{|c|}{$\begin{array}{l}\text { Note: All values are presented as number of patients unless otherwise noted. Only sensitive therapies considered as options (e.g., contraindicated therapies not considered } \\
\text { as an option). } \\
\text { aDoes not include } 1 \text { test that failed to show results. } \\
\text { bIncludes all patients enrolled in clinical trials with survival data available, regardless if CGP-directed or non-CGP-directed. } \\
\text { CGP = comprehensive genomic profiling; FDA =U.S. Food and Drug Administration; OS=overall survival. }\end{array}$} \\
\hline
\end{tabular}

In addition, an analysis of a separate cohort of patients who enrolled in clinical trials was undertaken to calculate the potential cost-benefit to the payer attributable to diversion of costs from the payer to the clinical trial sponsor. For this analysis, the medical record was reviewed to identify the treatment alternative to the clinical trials offered to the patient. When a specific alternative regimen was documented, that regimen was used in the analysis. For patients without a documented alternative regimen, one of the authors (Gribbin) determined the next best regimen. The monthly cost of each patient's alternate regimen was calculated based on the average sales price plus $6 \%$; costs were then multiplied by a progression-free survival (PFS) duration of 3.23 months, which was the median PFS reported for patients enrolled in phase 1 clinical trials in a meta-analysis of 346 studies by Schwaederle et al. (2016). ${ }^{13}$ All weight-based dosing used an assumption of an $80 \mathrm{~kg}$ person, and all therapies were assumed to have been administered using the FDA-approved dosing interval. Because payers (commercial and Medicare) typically continue to pay for routine care for beneficiaries enrolled in clinical trials, only drug therapies were considered in the cost diversion analysis, and all other costs of care were assumed to be paid by the payer, whether Medicare or commercial, after clinical trial enrollment.

This was a descriptive analysis, and no statistical tests were performed on the collected data.

\section{Results}

\section{Characteristics of Included Patients}

In total, 96 patients underwent CGP over the analysis period (Table 1). Median patient age at baseline was 63.5 years, and 46 were female. Almost all patients lived in a large central metropolitan ( $=51)$ or large fringe metropolitan ( $n=25)$ area. Between 2014 and 2016 (the years for which a full 12 months of data were available), the number of patients who underwent CGP testing each year ranged from 27 to 34 .

The most commonly tested cancer types were NSCLC $(n=36)$ and colorectal carcinoma $(n=13)$. Nearly all patients had stage IV disease $(n=78)$ at the time of CGP testing. Most patients had CGP testing before completion of first-line therapy $(n=51) ; 34$ patients had CGP testing following either first- or second-line treatment, and 11 patients had CGP testing following 3 or more lines of treatment.

The clinical and disease state requirements stated in the health plan CGP medical policy were met in 80 of 96 patients.

\section{Genomic Testing, Posttest Treatment, Clinical Trial Enrollment, and Outcomes}

Clinically relevant genomic alterations-defined as alterations that, on the basis of clinical or preclinical evidence, may be predictive for sensitivity or resistance to either FDA-approved or investigational therapies-were detected in 90\% of cases ( $\mathrm{n}=86 / 96$; Table 2 and Table 3). For 9 cases, no clinically 
Effect of a Collaboration Between a Health Plan, Oncology Practice, and Comprehensive Genomic Profiling Company from the Payer Perspective

FIGURE 1 Results of Pre-Index Conventional Molecular Diagnostic Testing and CGP

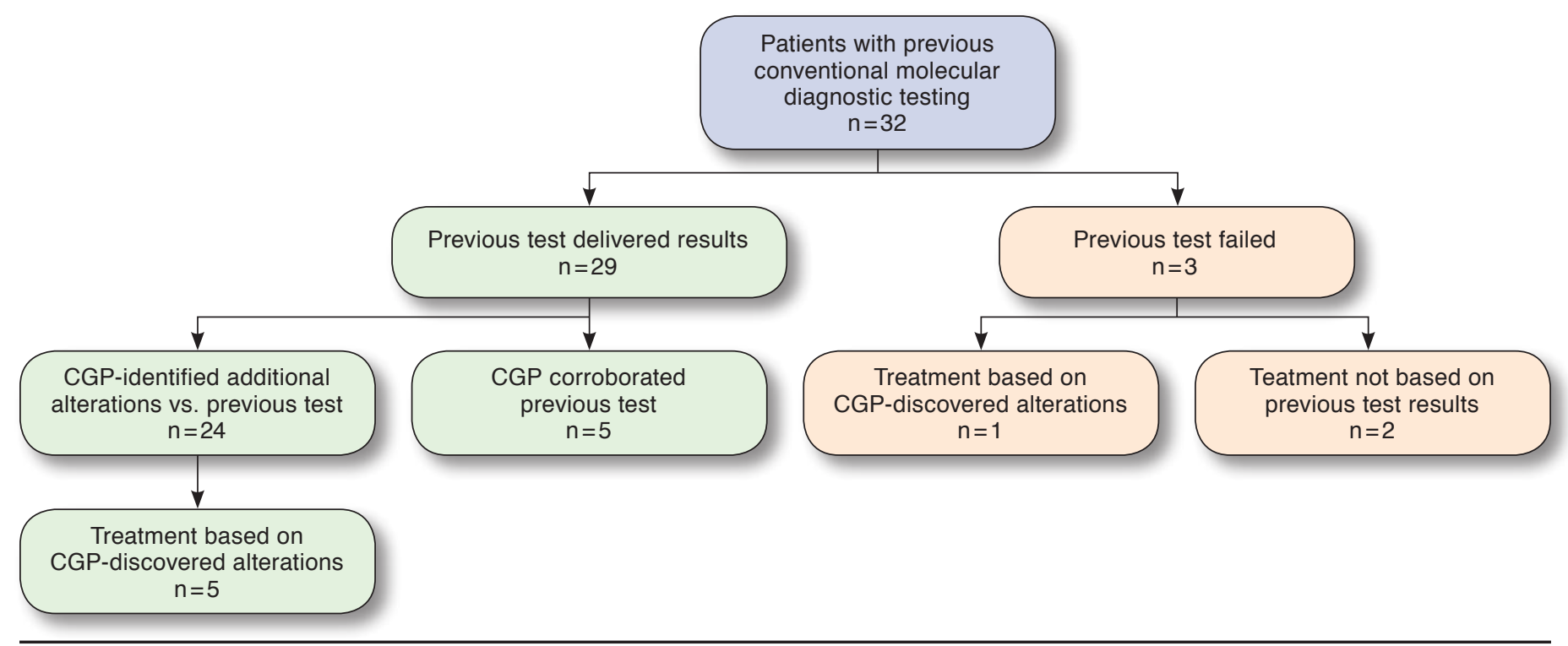

CGP = comprehensive genomic profiling.

relevant genomic alterations were detected by CGP. In 41 cases, an FDA-approved therapy for the treatment of the diagnosed tumor type (on-label) was associated with the CGP results based on clinical evidence, whereas in an additional 35 cases only an FDA-approved therapy in another tumor type (offlabel) was associated with the CGP results by clinical evidence. For an additional 10 cases, actionability was assessed at the clinical trial level only, because there were no FDA-approved therapies (on- or off-label) directly associated with their CGPdetected alterations. For these patients, at least 1 CGP-detected alteration provided rationale for enrollment in clinical trials for FDA-approved therapies (based on limited or emerging evidence) or for investigational therapies. A total of 24 patients declined treatment after CGP (Table 2).

Approximately half (33/70) of the patients who were treated following CGP received targeted therapy or immunotherapy. Of these 33 patients, 15 (45\%) received a therapy that matched a CGP-detected alteration or biomarker (Table 2 and Table 3). A total of 9 of the treated patients enrolled in clinical trials, and CGP results informed enrollment in at least 6 of these cases (Table 2). Twenty-one patients in the overall study population were treated with a CGP-matched targeted therapy or immunotherapy, or were enrolled in a clinical trial that was directly informed by CGP results (Table 3). This subset of patients represents approximately one third (21/64) of the patients who had 1 or more clinically relevant genomic alterations detected by CGP and who received any treatment following CGP testing.
Thirty-five patients experienced clinical benefit as assessed by the treating physician with the post-CGP treatment, and 57 experienced disease progression (Table 3). Among patients treated with CGP-matched targeted therapy or immunotherapy, 10 experienced clinical benefit and 5 experienced disease progression. Median OS after the index CGP test was 4.8 (mean 7.5 months, range 0-31) for the 67 patients for whom survival data were available. Median OS was 9.5 months (mean 9.5 months, range 1.1-24.2; $\mathrm{n}=10$ ) for patients treated with a CGP-matched therapy and 4.5 months (mean 8.5 months, range 2.3-20.6; $\mathrm{n}=7$ ) if enrolled in a clinical trial. For patients who were neither treated with a CGP-matched therapy nor enrolled in a clinical trial, median OS was 4.6 months (mean 6.9 months, range $0-30.9 ; \mathrm{n}=50$ ).

\section{Outcomes of CGP and Therapy in Patients with Previous Conventional Molecular Diagnostic Testing}

Records of previous conventional molecular diagnostic testing were found in 32 cases (Figure 1). Most of these cases $(n=21)$ had testing performed by an on-site pathology department or laboratory, with BRAF (B-Raf proto-oncogene, serine/ threonine kinase); ERBB2 (erb-b2 receptor tyrosine kinase 2); KRAS (KRAS proto-oncogene, GTPase); and EGFR (epidermal growth factor receptor) as the most commonly studied genes. Three of the 32 conventional molecular diagnostic tests failed, whereas 1 of 96 index CGP tests failed (10\% vs. 1\%). Among those who had previous conventional testing, CGP detected 


\begin{tabular}{l} 
TABLE 4 $\begin{array}{c}\text { Potential Drug Costs Diverted by } \\
20 \text { Patients Who Enrolled in Clinical } \\
\text { Trials After CGP }\end{array}$ \\
\hline
\end{tabular}

previously unidentified clinically relevant genomic alterations in $84 \%$ (27/32) of cases. Of these 27 cases with previous conventional testing, 6 (22\%) received treatment informed by the CGP-detected alterations that were not identified by previous testing, of whom 4 experienced clinical benefit (2 with initial clinical improvement followed by progression) from the CGP-directed treatment as reported by the treating physician (Appendix B, available in online article).

\section{Possible Cost Diversion from Clinical Trial Enrollment}

According to a separate analysis of 20 patients who enrolled in clinical trials following CGP, the payer may have accrued a total annual cost-benefit of approximately $\$ 500,000$ ( $\$ 25,000$ per patient) by the diversion of drug costs to the study sponsor, assuming a treatment duration of 3.23 months (Table 4). ${ }^{13}$

\section{Discussion}

This observational analysis characterizes the results of a medical policy implemented by a health plan that allowed for broad use of CGP testing in patients with advanced cancer (solid tumors, sarcomas, and hematologic malignancies) within a community oncology practice. Importantly, clinical and disease characteristic requirements in the medical policy were met in almost all patients (80 of 96). The findings from this analysis complement prospective analyses of CGP use in academic centers/clinical trials. ${ }^{18,31}$ CGP identified previously undetected and clinically relevant genomic alterations among most patients (27 of 32) with previous conventional molecular diagnostic testing. Nine patients had CGP results showing no associated therapies or available clinical trials, and 2 of these were enrolled in appropriate clinical trials based on the negative genomic findings. Of the 24 patients who remained untreated following CGP, over 90\% had clinically relevant genomic alterations detected. Although most patients were tested after 1 or 2 lines of therapy, many remained untreated, suggesting that early CGP testing may improve access to treatment options.

Given the recently released Centers for Medicare \& Medicaid Services (CMS) National Coverage Decision for NGS in patients with advanced cancer (which includes coverage for tests used in this observational analysis, as well as other qualifying tests), this observational analysis provides timely evidence of the utility of CGP in a real-world setting. ${ }^{32,33}$ The results presented here may provide insight into the clinical utility of broad CGP coverage for a commercial payer whose policy is in alignment with Medicare coverage of CGP in patients with advanced cancer. The high proportion of tested patients who met the health plan's medical policy clinical and disease requirements also suggests that CGP will be used in accordance with commercial payer medical policies if covered.

Importantly, the setting for this observational analysis was a community-based, nonacademic practice. Patients may prefer academic centers to community practices for clinical trial enrollment options and a perception of more advanced care opportunities. However, academic medical centers may be inconveniently located or too costly for some patients, and there is mixed evidence supporting the clinical and economic value of academic medical centers over community oncology practices. $^{34-38}$ Genomic tests inform the use of genomically matched and biomarker-driven therapies in patients with advanced cancer, including those available in clinical trials. Improved access to CGP may thus remove barriers to targeted treatment and clinical trial enrollment. Indeed, this approach of matching patients to investigational agents versus empirically chosen treatment has been shown efficacious in phase 1,2 , and 3 settings. This observational analysis demonstrates the feasibility of successful implementation of a payer medical policy and the effective use of CGP in a community oncology 
practice to optimally manage patients being considered for targeted and biomarker-driven therapies, including investigational therapies in clinical trials.

The observational analysis also found that covering CGP in a tumor-agnostic setting was not overly burdensome to the health plan's budget. In this payer-provider scenario, the estimated use rate was approximately 1 test per 10,000 health plan enrollees per year. A cost diversion analysis also demonstrated preliminary evidence of some cost-benefit to payers associated with CGP because clinical trial drug costs of patients would be paid by the study sponsor. The cost diversion estimate was conservative in that it did not incorporate medical costs. ${ }^{39}$ It also used a duration of treatment estimate based on overall PFS for all treatment arms in a previously published meta-analysis of phase 1 clinical trials; however, this same meta-analysis demonstrated that patients on genomically matched therapies have longer PFS, which could mean the actual cost offsets for the payer are even greater than estimated here. Using a treatment duration of 5.70 months, corresponding to the PFS associated with a personalized strategy, would yield a much higher cost diversion of $\$ 891,200$ in the present cohort. On the other hand, a treatment duration of 2.95 months (corresponding to a nonpersonalized treatment strategy), would have resulted in a lower cost diversion of $\$ 461,100$.

Finally, several cases in which patients received CGP after an initial conventional molecular diagnostic test illustrate the possible clinical value of CGP over conventional tests. For example, 1 patient with melanoma and a finding of high TMB from CGP was treated with pembrolizumab. Not only is pembrolizumab approved by the FDA for melanoma, but it may have been particularly suitable for this patient, since multiple clinical studies have demonstrated the efficacy of immunotherapies (including pembrolizumab) in patients with high TMB in several tumor types, including those with melanoma. . $5,40,41$ In a separate example, a patient with intraocular melanoma had an activating GNAQ ( $G$ protein subunit alpha q) alteration detected by CGP that had not been identified by an earlier hotspot test. The choice of trametinib (FDA approved and listed on the NCCN Drugs and Biologics Compendium for the treatment of BRAF V600 mutant melanoma) was based on phase 1 and phase 2 clinical trials demonstrating the efficacy of MEK (mitogen-activated protein kinase) inhibitors in patients with GNAQ mutant uveal melanoma. ${ }^{42-44}$ These patients, among others in the present observational analysis, benefited from the use of CGP by directing patients to alternative targeted therapies or immunotherapies that were not previously detected.

\section{Limitations}

There are limitations to the data presented here. This was a relatively small retrospective, observational analysis of patients with advanced cancer with no control group; however, the findings provide preliminary evidence that CGP detects additional and sometimes missed alterations compared with conventional molecular diagnostic tests and can potentially lead to treatment optimization. It is also notable that the patients included in this study are among the population supported for CGP use by the CMS and the FDA per the recent National Coverage Decision.

Changes in a patient's health insurance status or treating physician could have affected treatment choice, and medical records were not inclusive of all reasons for therapy choice. This is especially relevant in precision medicine, in which multiple therapeutic approaches may be available to a patient because of several clinically relevant genomic alterations associated with targeted therapies.

The OS outcomes we provided were limited to those patients with survival data available (67 of 96), and statistical methods such as Kaplan-Meier analysis were not applied to the data. Also, OS was determined with all tumor types grouped together without adjustment for survival inherent to different tumor types.

Finally, potential cost-savings data are exploratory and hypothesis generating; no formal economic modeling (e.g., budget impact) was conducted.

\section{Conclusions}

In this observational analysis of medical records, patients with advanced cancer received CGP at a community practice, enabled through a new coverage policy. For many patients, this led to treatment with targeted therapies and immunotherapies or enrollment in clinical trials based on their clinically relevant genomic alterations. The financial effect of introducing CGP to payers and oncology practices should be further explored, including the potential for cost offsets resulting from patient enrollment in clinical trials.

\section{Authors}

MITCHELL REITSMA, BA, MBA, Priority Health, Grand Rapids, Michigan, and Oakland University William Beaumont School of Medicine, Rochester, Michigan. JOHN FOX, MD, Priority Health, Grand Rapids, Michigan, and THOMAS E. GRIBBIN, MD, Cancer and Hematology Centers of West Michigan, Grand Rapids. PIERRE VANDEN BORRE, PhD; MATTHEW CAVANAUGH, BSN, RN; YAKOV CHUDNOVSKY, PhD; RACHEL L. ERLICH, PhD; and RACHEL ANHORN, PharmD, Foundation Medicine, Cambridge, Massachusetts.

AUTHOR CORRESPONDENCE: Rachel Anhorn, PharmD, Foundation Medicine, 150 Second St., Cambridge, MA 02141. E-mail: ranhorn@foundationmedicine.com. 


\section{Effect of a Collaboration Between a Health Plan, Oncology Practice, and Comprehensive Genomic Profiling Company from the Payer Perspective}

\section{DISCLOSURES}

No separate study-related funding was provided by or to Priority Health, Foundation Medicine, and Cancer and Hematology Centers of West Michigan. Data analysis by Reitsma was conducted as part of an internship funded by Priority Health. Reitsma and Fox are employed by Priority Health. Anhorn, Vanden Borre, Cavanaugh, Chudnovsky, and Erlich are employed by Foundation Medicine.

\section{ACKNOWLEDGMENTS}

The authors thank Bethany Sawchyn, PharmD, of Foundation Medicine, for providing a critical review of the manuscript, as well as Tariq I. Mughal, MD, formerly of Foundation Medicine, for contributions to earlier drafts. The authors also thank Marcia Reinhart, DPhil, and Tim Peoples, MA (employees of Analysis Group and funded by Foundation Medicine) for medical writing support.

\section{REFERENCES}

1. Cheng DT, Mitchell TN, Zehir A, et al. Memorial Sloan KetteringIntegrated Mutation Profiling of Actionable Cancer Targets (MSK-IMPACT): a hybridization capture-based next-generation sequencing clinical assay for solid tumor molecular oncology. J Mol Diagn. 2015;17(3):251-64.

2. Frampton GM, Fichtenholtz A, Otto GA, et al. Development and validation of a clinical cancer genomic profiling test based on massively parallel DNA sequencing. Nat Biotechnol. 2013;31(11):1023-31.

3. Pagès A, Foulon S, Zou Z, et al. The cost of molecular-guided therapy in oncology: a prospective cost study alongside the MOSCATO trial. Genet Med. 2017;19(6):683-90.

4. Suh JH, Johnson A, Albacker L, et al. Comprehensive genomic profiling facilitates implementation of the National Comprehensive Cancer Network Guidelines for lung cancer biomarker testing and identifies patients who may benefit from enrollment in mechanism-driven clinical trials. Oncologist. 2016;21(6):684-91.

5. Johnson DB, Dahlman KH, Knol J, et al. Enabling a genetically informed approach to cancer medicine: a retrospective evaluation of the impact of comprehensive tumor profiling using a targeted next-generation sequencing panel. Oncologist. 2014;19(6):616-22.

6. Amado RG, Wolf M, Peeters M, et al. Wild-type KRAS is required for panitumumab efficacy in patients with metastatic colorectal cancer. J Clin Oncol. 2008;26(10):1626-34

7. Mass RD, Press MF, Anderson S, et al. Evaluation of clinical outcomes according to HER2 detection by fluorescence in situ hybridization in women with metastatic breast cancer treated with trastuzumab. Clin Breast Cancer. 2005;6(3):240-46.

8. Perez EA, Romond EH, Suman VJ, et al. Trastuzumab plus adjuvant chemotherapy for human epidermal growth factor receptor 2-positive breast cancer: planned joint analysis of overall survival from NSABP B-31 and NCCTG N9831. J Clin Oncol. 2014;32(33):3744-52.

9. Kwak EL, Bang YJ, Camidge DR, et al. Anaplastic lymphoma kinase inhibition in non-small-cell lung cancer. N Engl J Med. 2010;363(18):1693-703.

10. Clark GM, Zborowski DM, Culbertson JL, et al. Clinical utility of epidermal growth factor receptor expression for selecting patients with advanced non-small cell lung cancer for treatment with erlotinib. J Thorac Oncol. 2006;1(8):837-46.

11. Tsao MS, Sakurada A, Cutz JC, et al. Erlotinib in lung cancer - molecular and clinical predictors of outcome. N Engl J Med. 2005;353(2):133-44.

12. Schwaederle M, Zhao M, Lee JJ, et al. Impact of precision medicine in diverse cancers: a meta-analysis of phase II clinical trials. J Clin Oncol. 2015;33(32):3817-25.
13. Schwaederle M, Zhao M, Lee JJ, et al. Association of biomarkerbased treatment strategies with response rates and progression-free survival in refractory malignant neoplasms: a meta-analysis. JAMA Oncol. 2016;2(11):1452-59

14. Gutierrez ME, Choi K, Lanman RB, et al. Genomic profiling of advanced non-small cell lung cancer in community settings: gaps and opportunities. Clin Lung Cancer. 2017;18(6):651-59.

15. Goodman AM, Kato S, Bazhenova L, et al. Tumor mutational burden as an independent predictor of response to immunotherapy in diverse cancers. Mol Cancer Ther. 2017;16(11):2598-608.

16. Rozenblum AB, Ilouze M, Dudnik E, et al. Clinical impact of hybrid capture-based next-generation sequencing on changes in treatment decisions in lung cancer. J Thorac Oncol. 2017;12(2):258-68.

17. Haslem DS, Van Norman SB, Fulde G, et al. A retrospective analysis of precision medicine outcomes in patients with advanced cancer reveals improved progression-free survival without increased health care costs. J Oncol Pract. 2017;13(2):e108-19.

18. Wheler JJ, Janku F, Naing A, et al. Cancer therapy directed by comprehensive genomic profiling: a single center study. Cancer Res. 2016;76(13):3690-701.

19. Hanna N, Johnson D, Temin S, et al. Systemic therapy for stage IV nonsmall-cell lung cancer: American Society of Clinical Oncology clinical practice guideline update. J Clin Oncol. 2017;35(30):3484-515.

20. National Cancer Institute. Cancer Moonshot Initiative Blue Ribbon Panel report 2016. October 17, 2016. Available at: https://www.cancer.gov/ research/key-initiatives/moonshot-cancer-initiative/blue-ribbon-panel/blueribbon-panel-report-2016.pdf. Accessed December 18, 2018.

21. National Comprehensive Cancer Network. Oncology research program. Available at: https://www.nccn.org/clinical_trials/clinicians.aspx. 2018. Accessed December 18, 2018.

22. Chow CJ, Habermann EB, Abraham A, et al. Does enrollment in cancer trials improve survival? J Am Coll Surg. 2013;216(4):774-80.

23. He J, Abdel-Wahab O, Nahas MK, et al. Integrated genomic DNA/ RNA profiling of hematologic malignancies in the clinical setting. Blood. 2016;127(24):3004-14.

24. Fox J. Lessons from an oncology medical home collaborative. Am J Manag Care. 2013;19(1 Spec No):SP5-9.

25. Kuntz G, Tozer JM, Snegosky J, Fox J, Neumann K. Michigan Oncology Medical Home Demonstration Project: first-year results. J Oncol Pract. 2014;10(5):294-97.

26. Priority Health. Multi-marker tumor panels. Medical policy 91609-R0. November 12, 2014. Revised November 2016. Available at: https://www. priorityhealth.com/provider/manual/auths/ /media/documents/medicalpolicies/91609.pdf. Accessed December 18, 2018.

27. Walker MS, Weinstein L, Luo R, Marino I. Pretreatment costs of care and time to initial treatment for patients with cancer of unknown primary. J Comp Eff Res. 2018;7(6):523-33.

28. Subbiah IM, Tsimberidou A, Subbiah V, Janku F, Roy-Chowdhuri S, Hong DS. Next generation sequencing of carcinoma of unknown primary reveals novel combinatorial strategies in a heterogeneous mutational landscape. Oncoscience. 2017;4(5-6):47-56.

29. Wei S, Lieberman D, Morrissette JJ, Baloch ZW, Roth DB, McGrath C. Using "residual" FNA rinse and body fluid specimens for next-generation sequencing: an institutional experience. Cancer Cytopathol. 2016;124(5):324-29.

30. Goodman AM, Choi M, Wieduwilt M, et al. Next generation sequencing reveals potentially actionable alterations in the majority of patients with lymphoid malignancies. JCO Precis Oncol. 2017;1(1):1-13.

31. Schwaederle M, Parker BA, Schwab RB, et al. Precision oncology: the UC San Diego Moores Cancer Center PREDICT experience. Mol Cancer Ther. 2016;15(4):743-52. 
Effect of a Collaboration Between a Health Plan, Oncology Practice, and Comprehensive Genomic Profiling Company from the Payer Perspective

32. U.S. Food and Drug Administration. FDA announces approval, CMS proposes coverage of first breakthrough-designated test to detect extensive number of cancer biomarkers. November 30, 2017. Available at: https:// www.fda.gov/NewsEvents/Newsroom/PressAnnouncements/ucm587273. htm. Accessed December 18, 2018.

33. Centers for Medicare \& Medicaid Services. Decision memo for next generation sequencing (NGS) for Medicare beneficiaries with advanced cancer (CAG-00450N). March 16, 2018. Available at: https://www.cms.gov/ medicare-coverage-database/details/nca-decision-memo.aspx?NCAId=290\& bc=AAAAAAAAQAAA\&. Accessed December 18, 2018.

34. Herring M, Huynh L, Duh MS, et al. Real-world treatment patterns in advanced pancreatic neuroendocrine tumors in the era of targeted therapy: perspectives from an academic tertiary center and community oncology practices. Med Oncol. 2017;34(5):88.

35. Rose TL, Deal AM, Basch E, et al. Neoadjuvant chemotherapy administration and time to cystectomy for muscle-invasive bladder cancer: an evaluation of transitions between academic and community settings. Urol Oncol. 2015;33(9):386.el-6

36. Veenstra CM, Epstein AJ, Liao K, et al. Hospital academic status and value of care for nonmetastatic colon cancer. J Oncol Pract. 2015;11(3):e304-12.

37. Lauseker M, Hasford J, Pfirrmann M, Hehlmann R, German CML Study Group. The impact of health care settings on survival time of patients with chronic myeloid leukemia. Blood. 2014;123(16):2494-96.
38. Cleeland CS, Mendoza TR, Wang XS, et al. Levels of symptom burden during chemotherapy for advanced lung cancer: differences between public hospitals and a tertiary cancer center. J Clin Oncol. 2011;29(21):2859-65.

39. Centers for Medicare \& Medicaid Services. National coverage determination (NCD) for routine costs in clinical trials (310.1). July 9, 2007. Available at: https://www.cms.gov/medicare-coverage-database/details/ncd-details.aspx?NC DId=1\&ncdver=2\&bc=AAAAgAAAAAAA\&. Accessed December 18, 2018.

40. Yarchoan M, Hopkins A, Jaffee EM. Tumor mutational burden and response rate to PD-1 inhibition. N Engl J Med. 2017;377(25):2500-01.

41. Johnson DB, Frampton GM, Rioth MJ, et al. Targeted next generation sequencing identifies markers of response to PD-1 blockade. Cancer Immunol Res. 2016;4(11):959-67.

42. Carvajal RD, Sosman JA, Quevedo JF, et al. Effect of selumetinib vs chemotherapy on progression-free survival in uveal melanoma: a randomized clinical trial. JAMA. 2014;311(23):2397-405.

43. Weekes CD, Von Hoff DD, Adjei AA, et al. Multicenter phase I trial of the mitogen-activated protein kinase 1/2 inhibitor BAY 86-9766 in patients with advanced cancer. Clin Cancer Res. 2013;19(5):1232-43.

44. Falchook GS, Lewis KD, Infante JR, et al. Activity of the oral MEK inhibitor trametinib in patients with advanced melanoma: a phase 1 doseescalation trial. Lancet Oncol. 2012;13(8):782-89. 
Effect of a Collaboration Between a Health Plan, Oncology Practice, and Comprehensive Genomic Profiling Company from the Payer Perspective

\section{APPENDIX A Priority Health Medical Policy on CGP Testing}

Eligible Conditions for Testing

- Patients newly diagnosed with stage IV NSCLC

- Patients newly diagnosed with cancer of unknown primary or primary anatomic site

- Patients with newly diagnosed hematologic malignancies with high frequencies of actionable mutations or limited treatment options in defined clinical care guidelines

- Patients for whom tissue to perform evidence-based tumor genome mutation analysis is not available

- Patients newly diagnosed with selected stage IV rare or uncommon solid tumors for whom very limited or no systemic treatment exists in clinical care guidelines or pathways

- Patients newly diagnosed with selected stage IV solid tumor types having poor prognosis, very limited benefit from standard of care chemotherapies, and a high prevalence of actionable genomic alterations

- Patients with stage IV solid tumors who have exhausted the established guideline-driven systemic therapy and requisite molecular testing but who desire further treatment

\section{Coverage Requirements for Matched Drugs}

To be covered, the prescribed drugs must meet 1 of the following 3 criteria:

- FDA-approved indication

- Listing in one of the following drug compendia:

- The American Hospital Formulary Service Drug Information

- Thomson Micromedex DrugDex or DrugPoints

- NCCN Guidelines

- Clinical Pharmacology

- Provider submission of at least 2 peer-reviewed journal articles

o whose primary purpose was to evaluate the use of the drug for the offlabel diagnosis for which it is requested; and

o that support the proposed off-label use as generally safe and effective for the patient's diagnosis.

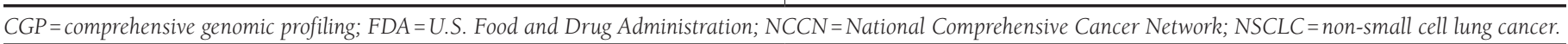


Effect of a Collaboration Between a Health Plan, Oncology Practice, and Comprehensive Genomic Profiling Company from the Payer Perspective

\section{APPENDIX B Description of Patients Who Received Treatment Informed by CGP-Detected Alterations that} Were Not Identified by Previous Conventional Molecular Diagnostic Testing

\begin{tabular}{l|c|c|c|c} 
Type of Cancer & Previous Test & $\begin{array}{c}\text { CGP-Detected Alteration or } \\
\text { Biomarker Used to Direct } \\
\text { Treatment }\end{array}$ & $\begin{array}{c}\text { CGP-Directed Treatments } \\
\text { Used or Clinical Trial } \\
\text { Enrollment }\end{array}$ & $\begin{array}{c}\text { Clinical Outcome Description } \\
\text { by Physician }\end{array}$ \\
\hline Invasive breast ductal carcinoma & Multigene panel & ERBB2 exon 20 insertion & Trastuzumab + paclitaxel & Clinical improvement \\
\hline Intraocular melanoma & BRAF (failed) & GNAQ 2209L & Trametinib & $\begin{array}{c}\text { Initial response followed by } \\
\text { disease progression }\end{array}$ \\
\hline Melanoma & BRAF & TMB-high & Pembrolizumab & Clinical improvement \\
\hline Colon adenocarcinoma & ALK, EGFR & BRAF V600E & $\begin{array}{c}\text { Oxaliplatin + capecitabine+ } \\
\text { bevacizumab (clinical trial) }\end{array}$ & $\begin{array}{c}\text { Clinical improvement followed by } \\
\text { minor disease progression }\end{array}$ \\
\hline Esophagus adenocarcinoma & ERBB2 & KRAS amplification & Trametinib & Disease progression \\
\hline Unknown primary melanoma & BRAF & GNAQ Q209P & Trametinib & Disease progression \\
\hline ALK
\end{tabular}

ALK=ALK receptor tyrosine kinase (gene name); BRAF = B-Raf proto-oncogene, serine/threonine kinase (gene name); CGP=comprehensive genomic profiling; EGFR=epidermal growth factor receptor (gene name); ERBB2 =erb-b2 receptor tyrosine kinase 2 (gene name); GNAQ=G protein subunit alpha $q$ (gene name); $I D=$ identification; KRAS=KRAS proto-oncogene, GTPase (gene name); TMB=tumor mutational burden. 